\title{
Dengue Virus Detection by Using Reverse Transcription- Polymerase Chain Reaction in Saliva and Progeny of Experimentally Infected Aedes albopictus from Brazil
}

\author{
Márcia Gonçalves de Castro/ ${ }^{+}$, Rita Maria Ribeiro Nogueira*, \\ Hermann Gonçalves Schatzmayr*, Marize Pereira Miagostovich*, \\ Ricardo Lourenço-de-O liveira
}

\author{
Laboratório de Transmissores de Hematozoários *Laboratório de Flavivírus, Instituto Oswaldo Cruz-Fiocruz, Av. Brasil 4365, \\ 21045-900 Rio de Janeiro, RJ, Brasil
}

Oral susceptibility and vertical transmission of dengue virus type 2 (DENV-2) in an Aedes albopictus sample from Rio de Janeiro was estimated. The infection (36.7\%) and transmission (83.3\%) rates for Ae. albopictus were higher than those of an Ae. aegypti colony used as control, 32.8 and 60\%, respectively. Fourth instar larvae and females descendants of 48.5 and $39.1 \%$ of experimentally infected Ae. albopictus showed to harbor the virus. The oral susceptibility and the high capacity to assure vertical transmission exhibited by Ae. albopictus from Brazil reinforce that this species may play a role in the maintenance of the virus in nature and be a threat for dengue control in the country.

Key words: Aedes albopictus - dengue virus type 2 - vector competence - vertical transmission - Brazil

Dengue has been considered the most important arboviruses affecting man (Gubler 1998). In Brazil, epidemics have been reported since the early 1980's, few years after the re-colonization of the country by Aedes aegypti. Currently, Ae. aegypti is spread in Brazil and three dengue virus (DENV) serotypes (DENV-1, DENV-2, DENV-3) co-circulate in 25 out of 27 Brazilian states. So far DENV4 has not been reported in Brazil since 1982 (Schatzmayr 2000, da Silva Junior et al. 2002).

Ae. albopictus, an important dengue vector in Asia (Shroyer 1986) and the primary vector in a recent outbreak in Hawaii (MNWR 2002), was discovered in Brazil in 1986. The species is considerably spread in the country, especially in the South and Southeast regions (Santos 2003). The Brazilian samples of Ae. albopictus have proved to be experimentally susceptible to both DENV and yellow fever virus (Miller \& Ballinger 1988, Lourenço-deOliveira et al. 2003). However, the potential of Ae. albopictus populations occurring in Brazil as dengue vector is far to be known. This is specially because the evidence that successive introductions and establishment of Ae. albopictus populations from other geographic regions go on occurring that may lead to genetic modification and consequent changes in vector competence and transmission patterns (Lourenço-de-Oliveira et al. 2003, Lounibos et al. 2003).

Vector competence to DENV depends on the susceptibility of a mosquito to become infected and subsequently

${ }^{+}$Corresponding author. Fax: +55-21-2573.4468. E-mail: mcastro@ioc.fiocruz.br

Received 30 August 2004

Accepted 20 October 2004 transmit the virus through the bite. For that, ingested virus particles during a blood meal on an infected host must reach the epithelium of the midgut, replicate, and pass through this main barrier to get into the haemocell. Then, virus replication and dissemination take place cell-to-cell all over the mosquito's body and finally the virus reach the epithelium of the salivary gland and may be transmitted by the injection of infected saliva in a susceptible vertebrate host (Gubler \& Kuno 1997). Arboviruses may also be transmitted among mosquitoes in other ways: venereal and vertical transmission, i.e. when the virus is transmitted to the progeny of an infected female (Woodring et al. 1996).

DENV-1 was once detected in immatures of Ae. albopictus collected in Southeastern Brazil (Serufo et al. 1993) and DENV-2 and DENV-3 were isolated from males caught in Mexico (Ibáñez-Bernal et al. 1997), thus giving evidences that vertical transmission in this species may be an important event for the maintenance of the virus in nature in the Americas.

In this manuscript, the oral susceptibility and the vertical transmission of DENV-2 are experimentally evaluated for a Brazilian Ae. albopictus sample and the potential role of this mosquito species in the epidemiology of dengue in the country is discussed.

\section{MATERIALS AND METHODS}

Virus strain - The DENV-2 strain 63139 (collection of the Flavivirus Laboratory, Instituto Oswaldo Cruz) was isolated in 1998 from a dengue fever patient's sera from Rio de Janeiro by inoculation into Ae. albopictus C6/36 cell line and was identified by immunofluorescence using type-specific monoclonal antibodies (Igarashi 1978, Gubler et al. 1984). The DENV-2 stocks were obtained by the passage in Ae. albopictus clone C6/36 culture cells and were used at a titer of $10^{6}$ TCID $_{50}$ per $0.1 \mathrm{ml}($ Reed \& Muench 1938). 
Mosquito experimental infections - Seven assays of mosquito infections with DENV-2 were conducted using a laboratory open colony of Ae. albopictus established from specimens collected in Jacarepaguá, Rio de Janeiro, one year before. Dozens of offsprings (F1) of wild Ae. albopictus females collected in the same site were bimonthly introduced in the colony. The Manguinhos colony of Ae. aegypti, derived from larvae collected in Rio de Janeiro in 1987, was used as control of virus infectivity.

Sets of 150 5-7 days old females were starved $24 \mathrm{~h}$ before infection. The infectious meal contained $1 / 3$ washed sheep erythrocytes, $1 / 3$ virus suspension and $1 / 3$ fetal calf serum (FCS, Gibco-Brl) containing 10\% sucrose, according to Gubler and Rosen (1976). After a blood meal of $2 \mathrm{~h}$, completely engorged females were kept at $27^{\circ} \mathrm{C}$ and $70-80 \%$ humidity, and daily provided with $10 \%$ sucrose. On the 7th day after taking the infectious blood meal, a non-infectious blood meal (1/3 sheep erythrocytes, $1 / 3$ Leibovitz L-15 culture medium and 1/3 FCS) was offered to mosquitoes. On the 10th day, mosquitoes were individually transferred to vials with wet filter paper on the bottom as oviposition support. On day 14th, mosquitoes were frozen $\left(-70^{\circ} \mathrm{C}\right)$. Mosquito eggs were kept at $27^{\circ} \mathrm{C}$ and $70-80 \%$ air humidity on wet filter paper for 10 days and let to dry from day 11 to 20 after oviposition. Egg batches of each female were immerged in tap water on the 21 st day. Mosquitoes were reared in controlled conditions of temperature and humidity $\left(27^{\circ} \mathrm{C}, 70-80 \%\right)$, according to Consoli and Lourenço-de-Oliveira (1994). Samples of fourth instar larvae and emerged adult females from each egg batch were pooled according to stage and sex and frozen $\left(-70^{\circ} \mathrm{C}\right)$ for subsequent virus isolation.

We used indirect immunofluorescence assay (IFA) on head squashes of mosquitoes randomly selected in each set to determine infection rates. Individual head squashes were let to dry and fixed with acetone at $-20^{\circ} \mathrm{C}$, for $20 \mathrm{~min}$. Fluorescein isothiocyanate-conjugated anti mouse $\operatorname{IgG}$ and monoclonal antibody $(3 \mathrm{H} 5$, gently provided by $\mathrm{D}$ Gubler, CDC), diluted 1:20, was used to detect DENV antigens in nervous tissues in head of assayed mosquitoes. Carcasses of these mosquitoes were individually ground in Leibovitz-15 culture medium (Sigma) and centrifuged (3000 rpm at $4^{\circ} \mathrm{C}$, for $15 \mathrm{~min}$ ); the supernatant was inoculated in C6/36 cell culture (Igarashi 1978) for virus re-isolation. Carcasses of the reminder mosquitoes fed with the infectious meal in each set were pooled and inoculated in $\mathrm{C} 6 / 36$ cell. Cell cultures were incubated at $28^{\circ} \mathrm{C}$ for 14 days, when virus was detected by IFA.

$R N A$ extraction and reverse transcriptase-PCR amplification (RT-PCR) - Viral RNA was extracted from the supernatant of infected cells by using the QIAmp Viral Mini Kit (Qiagen, Inc., Valencia, CA) according to the manufacturer's protocol. RT-PCR for detecting DENV was performed according to Lanciotti et al. (1992). Amplification was conducted in $0.5 \mathrm{ml}$ Eppendorf ${ }^{\mathrm{TM}}$ tubes using a Model 2400 thermocycler (Perkin-Elmer, Norwalk, CT) or PTC-200-60 thermocycler (MJ Research, Inc., Watertown, MA). Negative controls were included as follows: saliva of non infected Ae. albopictus and Ae. aegypti from laboratory colonies, and water. DENV-2 from C6/36 cell culture was the positive control.

Detection of virus and viral RNA in mosquito saliva To evaluate virus transmission rates (no. transmitting/ no. of infected mosquitoes) we searched DENV-2 in the saliva of $52 \mathrm{Ae}$. albopictus and $52 \mathrm{Ae}$. aegypti randomly selected females which were induced to salivate for 15 min in capillary tubes containing $4 \mu \mathrm{l}$ mineral oil on day 14th after taken the infectious meal, as described by Hurlbur (1966). The mineral oil containing each sample of mosquito saliva was dropped into $20 \mu \mathrm{l}$ of $\mathrm{NaCl} 0.85 \%$ and centrifuged (1500 rpm, per $3 \mathrm{~min}$ ). $130 \mu \mathrm{l}$ of PBS pH 7.2 were added to $10 \mu 1$ of supernatant of mosquito saliva sample to a final volume of $140 \mu \mathrm{l}$ for the detection of viral RNA by RT-PCR.

\section{RESULTS}

Infection rates, as determined by IFA in head squashes, were $36.7 \%$ for Ae. albopictus from Rio de Janeiro, and $32.8 \%$ for the control Ae. aegypti. Virus re-isolation in C6/ 36 cell culture from respective mosquito carcasses was achieved in $35.9 \%$ of Ae. albopictus and $26.7 \%$ of Ae. aegypti engorged females. Mortality rate was higher in Ae. albopictus (43.9\%) than in Ae. aegypti (29.6\%) (Table I).

Virus transmission rate for Ae. albopictus, as determined by the presence of viral RNA in the saliva of infected females, was higher than the infection rate (Table I). Viral RNA was detected in the saliva of $48.1 \%$ (25/52) of Ae. albopictus females on the 14th day after the infectious blood meal. In $16.7 \%$ of cases (5/30) viral RNA was not detected in the saliva of infected Ae. albopictus fe-

TABLE I

Aedes albopictus and Ae. aegypti females infected with dengue virus type 2 according to tissue and method used for virus detection, and transmission and mortality rates, on the 14th day after an infectious blood meal

\begin{tabular}{lccccc}
\hline Mosquito species & $\begin{array}{c}\text { Head squash } \\
\text { IFA } \\
\text { pos/tested }\end{array}$ & $\begin{array}{c}\text { Carcass } \\
\text { Cell culture } \\
\text { pos/tested }\end{array}$ & $\begin{array}{c}\text { Saliva } \\
\text { RT-PCR } \\
\text { pos/tested }\end{array}$ & $\begin{array}{c}\text { Transmission rate } \\
\text { no. transmitting/ } \\
\text { no. infected }\end{array}$ & $\begin{array}{c}\text { Mortality } \\
\text { rate } \\
\text { dead/tested }\end{array}$ \\
\hline Ae. albopictus & $22 / 60$ & $37 / 103$ & $25 / 52$ & $25 / 30$ & $186 / 423$ \\
Ae. aegypti & $36.7 \%$ & $35.9 \%$ & $48.1 \%$ & $83.3 \%$ & $43.9 \%$ \\
& $19 / 58$ & $27 / 101$ & $12 / 52$ & $12 / 20$ & $100 / 338$ \\
& $32.8 \%$ & $26.7 \%$ & $23.1 \%$ & $60 \%$ & $29.6 \%$ \\
\hline
\end{tabular}

IFA: indirect immunofluorescence; Cell culture: inoculation in Ae. albopictus C6/36 cell culture; RT-PCR: reverse transcriptasepolymerase chain reaction 
males, as confirmed by inoculation of respective carcasses in cell culture. Thus, $83.3 \%$ of infected Ae. albopictus was competent to transmit the virus on the 14th day after a blood meal containing DENV-2. Only $23.1 \%$ of Ae. aegypti had positive saliva (Table I), and viral RNA was not detected in the saliva of 8 out of $20(20 \%)$ females whose carcass harbored DENV-2. Thus, the transmission rate for Ae. aegypti was $60 \%$.

We obtain 1318 eggs from Ae. albopictus females between the 10th and 14th days after they have taken the blood meal containing dengue virus. Only 468 of the derived larvae reached the 4th instar, and most of them $(\mathrm{N}=$ 284) were pooled and frozen for subsequent virus detection and the remainder were reared until adult (Table II). A total of 39 pools comprising 2 to 10 larvae was inoculated in cell culture. Virus was isolated from pooled larvae descendants of $48.5 \%$ (16/33) of infected females. Vertical transmission was confirmed by DENV-2 isolation from pooled female ( 25 examined pools of 2 to 9 females) descendants of $39.1 \%$ of experimentally infected Ae. albopictus (Table II).

A total of 2003 eggs was laid by the control Ae. aegypti that had an infectious blood meal. From 638 descendant larvae that reached the 4th instar, 521 were pooled (37 pools comprising 4 to 23 larvae) inoculated in cell culture (Table II), resulting in the isolation of virus from the progenies of $27.3 \%$ (9/33) of infected females. Infection rates were lower when virus was searched in adults descendant from Ae. aegypti fed on with a blood meal containing DENV-2: virus was isolated from $13.6 \%(3 / 22)$ of pooled females offspring of infected Ae. aegypti.
TABLE II

Dengue virus isolation from offsprings of Aedes aegypti and Ae. albopictus females which parental generation has taken an infectious blood meal containing dengue virus type 2 (DENV-2) according to development stages

\begin{tabular}{ll}
\hline & \multicolumn{2}{c}{ Mosquito species } \\
\cline { 2 - 3 } Offsprings & Ae.albopictus aegypti
\end{tabular}

4th instar larvae

$\mathrm{Nr}$ of examined specimens 284 (39 pools) 521 (37 pools)

positive pools $\quad 46.2 \%$

$(18 / 39)$

infected offsprings $\quad 48.5 \%$

$(16 / 33)$

$32.4 \%$

$(12 / 37)$

$27.3 \%$

$(9 / 33)$

Adult females

Nr of examined specimens 92 (25 pools) 59 (22 pools) positive pools

$36 \%$

$(9 / 25)$

$13.6 \%$

$(3 / 22)$

infected offsprings

$39.1 \%$

$(9 / 23)$

$13.6 \%$

$(3 / 22)$

\section{DISCUSSION}

The assayed Ae. albopictus sample from Rio de Janeiro showed to be susceptible to oral infection by DENV-2, being more competent to transmit the virus both horizontally and vertically than the Manguinhos Ae. aegypti colony. It was demonstrated that (1) the infection rate for Ae. albopictus was higher than for the Ae. aegypti

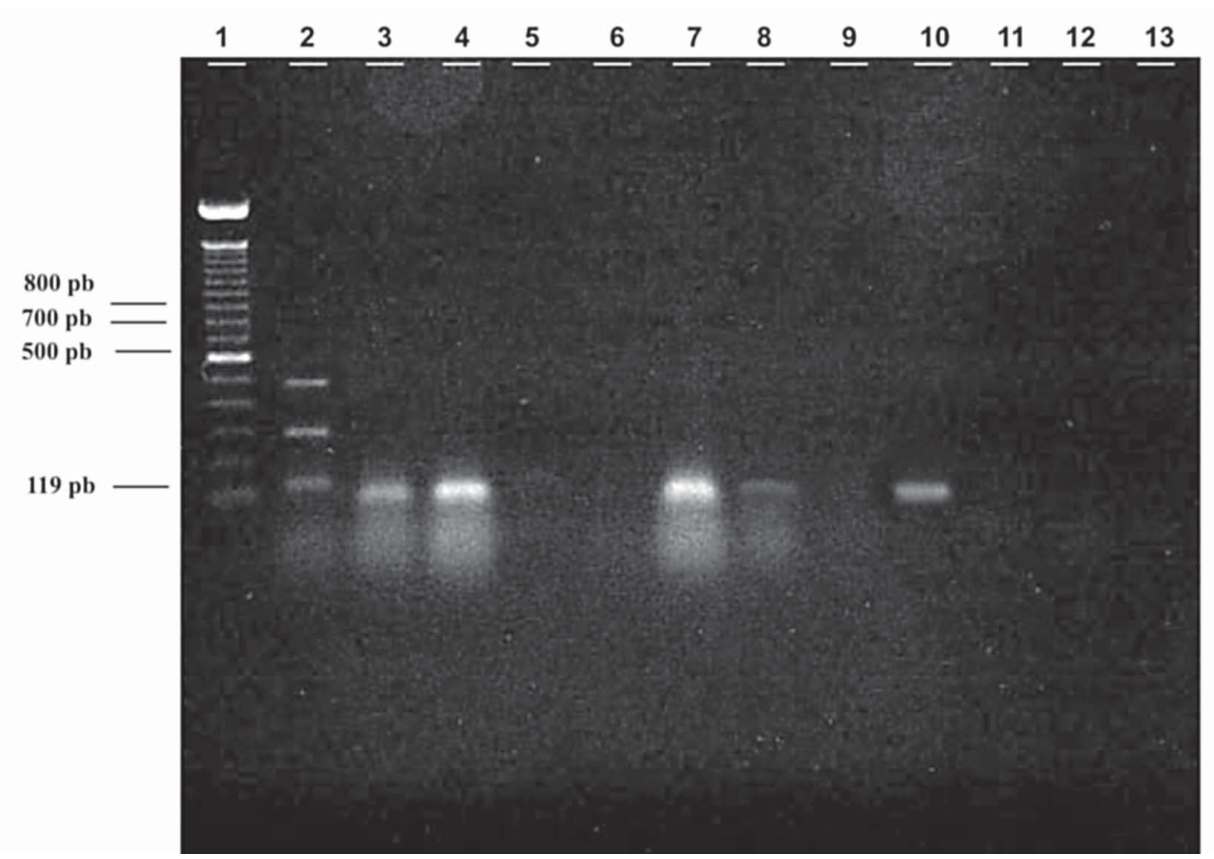

Reverse transcriptase-polymerase chain reaction (RT-PCR) profiles of dengue virus type 2 (DENV-2) detected in mosquito saliva. Ethidium bromide stained 1\% agarose gel. Lanes - 1: molecular weight marker 100bp (Gibco); 2: molecular weight marker of DENV-1, DENV-2, and DENV-3 RT-PCR products corresponding to $482 \mathrm{bp}, 119 \mathrm{bp}$, and $290 \mathrm{bp}$, respectively; 3 to 9: saliva of Aedes albopictus females on the 14th day after taking an infectious blood meal containing DENV-2;3, 4, 7, and 8: DENV-2 infected saliva of experimental Ae. albopictus; 5, 6, and 9: DENV-2 non-infected saliva of experimental Ae. albopictus; 10: positive control, DENV-2 isolated from C6/ 36 cell culture; lanes 11 to 13 negative controls; 11: water; 12, 13: saliva of non-infected Ae. aegypti and Ae. albopictus females from laboratory colonies. 
control colony; (2) the majority ( $83.3 \%$ ) of Ae. albopictus females in which the infection by DENV-2 was established, the viral RNA was detected in the saliva on the 14th day after a blood meal containing the virus; and (3) the virus was transmitted to females descendants of nearly $40 \%$ of the experimentally infected Ae. albopictus.

The infection rate for DENV-2 we assessed for a colony of Ae. albopictus from Rio de Janeiro (36.7\%) was similar to that found by Miller and Ballinger (1988) for a colony of the same species from Cariacica (38\%), a site also located in Southeastern Brazil. Nevertheless, other Brazilian samples of Ae. albopictus have proved to be more efficient in replicating DENV-2 than the above mentioned. When analyzing F1 generation of 10 Brazilian samples of Ae. albopictus, Lourenço-de-Oliveira et al. (2003) estimated that infection rates for dengue were mostly between 50 and $80 \%$, although infection rates have varied from 22.5 to $71.4 \%$ in four of their samples from Rio de Janeiro. These divergences are probably due to differences in experimental conditions, specially the number of generations of mosquitoes used, the method of virus titration and virus titer in the infectious blood meal, as demonstrated before (Vazeille et al. 2002). Indeed, Lourenço-deOliveira et al. (2003) used a distinct method of virus titration and a much higher virus titer $\left(10^{8.2}\right.$ MID50; where MID50 is $50 \%$ mosquito infectious dose) than ours $\left(10^{6}\right.$ TCD50) for mosquito infection. Other authors have used DENV titer similar to ours in the infectious blood meal of mosquitoes (Gubler \& Rosen 1976, 1977, Sunarto et al. 1979, Rosen et al. 1985).

We offer the DENV-2 infectious blood meal to mosquitoes for a period of $2 \mathrm{~h}$, as did several previous authors (e.g., Gubler \& Rosen 1976, Gubler et al. 1979, Rosen et al. 1985). However, we noticed that virus titer in the experimental blood meal dropped considerably during that period of mosquito repast. In our experiments, DENV-2 titer was around $10^{6}$ TCD50 in the first $10 \mathrm{~min}$ of the blood meal, whereas it ranged from $10^{4.8}$ TCID50 to $10^{2.0}$ TCD50 in $2 \mathrm{~h}$. The infection and transmission rates for DENV in a susceptible mosquito population also depend on the amount of ingested infectious virus particles. Therefore, mosquitoes that promptly imbibed the infectious blood meal have much more chance to become infected than those engorged $2 \mathrm{~h}$ latter, since the formers ingest a much higher number of infectious viruses. Thus, short periods for mosquito engorgements (such as 10 to $20 \mathrm{~min}$ ) are recommended to avoid false heterogeneous dengue infection rates for a single mosquito sample, such as has already been done by some authors (e.g., Johnson et al. 2002, Lourenço-de-Oliveira et al. 2002, 2003, Vazeille et al. 2002). This data also highlight the importance of standardizing procedures such as the duration of mosquito experimental infectious blood meal to estimate infection rate.

The presence of DENV-2 in samples of saliva of assayed Ae. albopictus and the control was assessed essentially by RT-PCR, although some samples were also inoculated in C6/36 cell cultures with poor results. DENV2 was re-isolated in cell culture only in respectively zero and $12.5 \%$ of Ae. aegypti and Ae. albopictus saliva samples in which viral RNA was detected by RT-PCR. On the other hand, DENV-2 specific fragments were generated by RT-PCR in very diluted minute drops of $80 \%$ of saliva samples of Ae. albopictus females whose head squashes revealed DENV-2 antigens by IFA. Thus RTPCR is an useful, specific and sensible method for the detection of DENV in saliva of mosquitoes. Indeed, RTPCR has proved to be an efficient method for diagnose of DENV in human sera and blood with very low viral titers (Lanciotti et al. 1992). This technique allowed us to demonstrate that the bite of $48 \%$ of the tested Ae. albopictus females from Rio de Janeiro were infected with DENV-2 on the 14th day after taken a artificial blood meal containing the virus.

Vertical transmission of DENV-2 was demonstrated to occur in almost half (16/33) of experimentally infected $A e$. albopictus, when considering virus isolation from pooled 4th instar larvae derived from their offspring. The frequency of vertical transmission diminished among females descendants of infected Ae. albopictus. Accordingly, virus was isolated in pooled females descendants of $39.1 \%$ of orally infected Ae. albopictus females. On the other hand, this rate is even higher than that we obtained for infected Ae. aegypti (13.6\%). These rates are in accordance or sometimes higher than those found by Rosen et al. (1983), for both Ae. albopictus and Ae. aegypti fed with blood containing DENV-1 and DENV-2, and by Bosio et al. (1992) and Gokhale et al. (2001) for Ae. albopictus.

Cumulated mortality at 14 day of orally infected $A e$. albopictus (43.9\%) and Ae. aegypti (29.6\%) (Table I) was in accordance with the literature for both dengue infected and non-infected mosquitoes (Vazeille-Falcoz et al. 1999). On the other hand, survivorship of vertically infected larval batches and/or fecundity of parental generation seemed to be affected by dengue infection, since the number of descendant larvae that reached the 4th instar was considerably smaller than that of laid eggs. We obtain 1318 eggs from Ae. albopictus females that have taken an infectious blood meal but only 468 derived larvae reached the 4th instar. A total of 2003 eggs was laid by Ae. aegypti, but only 638 descendant larvae reached the 4th instar. Joshi et al. (2002) observed a longer duration in larval stages and diminishing in fecundity and fertility of the DENV-3 transovarially infected batches of experimental Ae. aegypti. Notwithstanding, these authors demonstrated that DENV persisted in successive generations of $A e$. aegypti infected through vertical transmission and suggested that mosquitoes may play an important role in the maintenance of dengue virus in nature such as a reservoir.

Since Ae. albopictus samples from Rio de Janeiro and other southeastern sites have demonstrated to be susceptible to DENV and exhibited a high capacity to ensure vertical transmission, as evidenced herein and by previous workers (Mitchell \& Miller 1990, Lourenço-de-Oliveira et al. 2003), we may presume that this species may play a role in the maintenance of the virus transmission in this important entry point of both DENV and new mosquito populations in Brazil (Schatzmayr 2000, Luz et al. 2003, Lourenço-de-Oliveira et al. 2004) and that the expansion of this mosquito is a threat for dengue control in the country so far neglected by the nation campaigns. In the en- 
demic great metropolitan area of Rio de Janeiro, where species segregation has shown that Ae. albopictus predominate over Ae. aegypti in suburban and semi-rural neighborhoods (Braks et al. 2003) and no specific effort is made to control Ae. albopictus, this species may play an important role in the epidemiology of dengue by undertaking a continuing silent transmission of virus in such zones during inter-epidemic periods, specially via vertical transmission. Furthermore, dengue transmission patterns in some Brazilian areas may modify as an outcome of changing in population genetic composition of Ae. albopictus due to the underway invasion of new populations of this species in the American continent (Birungi \& Munstermann 2002, Lourenço-de-Oliveira et al. 2003, Lounibos et al. 2003). Therefore Ae. albopictus vector capacity for DENV types circulating in the continent needs to be continuously estimated.

\section{ACKNOWLEDGEMENTS}

To José da Costa Farias, José de Carvalho Filho, and Marcelo Quintela Gomes for the technical support. To Maria Goreti Rosa-Freitas for the critical reviewing of the manuscript.

\section{REFERENCES}

Birungi J, Munstermann LE 2002. Genetic structure of Aedes albopictus (Diptera: Culicidae) populations based on mitochondrial ND5 sequences: evidence for an independent invasion into Brazil and United States. Ann Entomol Soc Am 95: 125-132.

Bosio CF, Thomas RE, Grimstad PR, Rai KS 1992. Variation in the efficiency of vertical transmission of dengue-1 virus by strains of Aedes albopictus (Diptera: Culicidae). J Med Entomol 29: 985-989.

Braks M, Honório NA, Lourenço-de-Oliveira R, Juliano SA, Lounibus PL 2003. Convergent habitat segregation of Aedes aegypti and Aedes albopictus (Diptera: Culicidae) in southeastern Brazil and Florida, USA. J Med Entomol 40: 785794.

Consoli RAGB, Lourenço-de-Oliveira R 1994. Principais Mosquitos de Importância Sanitária no Brasil, Editora Fiocruz, Rio de Janeiro, $225 \mathrm{pp}$.

Da Silva Junior JB, Siqueira Junior JB, Coelho GE, Vilarinhos PTR, Pimenta Junior FG 2002. Dengue in Brazil: current situation and prevention and control activities. Epidemiol Bull 23: 3-6.

Gokhale MD, Barde PV, Sapkal GN, Gore MM, Mourya DT 2001. Vertical transmission of dengue-2 virus through Aedes albopictus mosquitoes. J Commun Dis 33: 212-215.

Gubler DJ 1998. Resurgent vector-borne diseases as a globalhealth problem. Emerg Infec Dis 4: 442-449.

Gubler DJ, Kuno G 1997. Dengue and Dengue Hemorrhagic Fever, CAB International, New York, 478 pp.

Gubler DJ, Rosen L 1976. Variation among geographic strains of Aedes albopictus in susceptibility to infection with dengue viruses. Am J Trop Med Hyg 25: 318-25.

Gubler DJ, Rosen L 1977. Quantitative aspects of replication of dengue viruses in Aedes albopictus (Diptera: Culicidae) after oral and parenteral infection. J Med Entomol 13: 469472.

Gubler DJ, Nalim S, Tan R, Saipan H, Saroso-Sulianti J 1979. Variation in susceptibility to oral infection with dengue viruses among geographic strains of Aedes aegypti. Am J Trop Med Hyg 28: 1045-1052.

Gubler DJ, Kuno G, Sather GE, Velez M, Oliver A 1984. Use of mosquito cell cultures and specific monoclonal antibodies in surveillance for dengue virus. Am J Trop Med Hyg 33: $158-165$.

Hurlbur HS 1966. Mosquito salivation and virus transmission. Am J Trop Med Hyg 15: 989-999.

Ibáñez-Bernal S, Briceño B, Mutebi SP, Argot E, Argot E, Rodríguez G, Martínez-Campos C, Paz R, De la FuenteSan Román P, Tapia-conyer R, Fliner A 1997. First record in America of Aedes albopictus naturally infected with dengue virus during the 1995 outbreak at Reynosa, Mexico. Med Vet Entomol 11: 305-309.

Igarashi A 1978. Isolation of a Singh's Aedes albopictus cell clone sensitive to dengue and chikungunya viruses. J Gen Virol 40: 531-44.

Johnson BA, Chambers TV, Crabtree MB, Bhatt TR, Guirakhoo F, Monath TP, Miller BR 2002. Growth characteristics of chimerivaxTM- DEN 2 vaccine virus in Aedes aegypti and Aedes albopictus mosquitoes. Am J Trop Med Hyg 67: 260265.

Joshi V, Mourya DT, Sharma RC 2002. Persistence of dengue3 virus through transovarial transmission passage in successive generations of Aedes aegypti mosquitoes. Am J Trop Med Hyg 67: 158-61.

Lanciotti RS, Calisher CH, Gubler DJ, Chang GJ, Vorndam V 1992. Rapid detection and typing of dengue viruses from clinical samples by using reverse transcriptase-polymerase chain reaction. J Clin Microbiol 30: 545-551.

Lounibos LP, Escher RL, Lourenço-de-Oliveira R 2003. Assimetric evolution of invasive diapause in temperate and tropical invasive populations of Aedes albopictus (Ditera: Culicidae). Ann Entomol Soc Am 96: 512-518.

Lourenço-de-Oliveira R, Vazeille M, Filippis AMB, Failloux AB 2002. Oral susceptibility to yellow fever virus of Aedes aegypti from Brazil. Mem Inst Oswaldo Cruz 97: 437-439.

Lourenço-de-Oliveira R, Vazeille M, Filippis AMB, Failloux AB 2003. Aedes albopictus from Brazil and southern United States: genetic variation and vector competence for dengue and yellow fever viruses. Am J Trop Med Hyg 69: 105-114.

Lourenço-de-Oliveira R, Vazeille M, Filippis AMB, Failloux AB 2004. Aedes aegypti in Brazil: genetically differentiated populations with high susceptibility to dengue and yellow viruses. Trans R Soc Trop Med Hyg 98: 43-54.

Luz PM, Codeço CT, Massad E, Struchiner CJ 2003. Uncertainties regarding dengue modeling in Rio de Janeiro, Brazil. Mem Inst Oswaldo Cruz 98: 871-878.

Miller BR, Ballinger ME 1988. Aedes albopictus mosquitoes introduced into Brazil: vector competence for yellow fever and dengue viruses. Trans $R$ Soc Trop Med Hyg 82: 476477.

Mitchell CJ, Miller BR 1990. Vertical transmission of dengue viruses by strains of Aedes albopictus recently introduced into Brazil. J Am Mosq Control Assoc 6: 251-253.

MMWR-Mortality and Morbidity Weekly Reports 2002. Editorial Note. CDC 51: 281-286.

Reed LJ, Muench H 1938. A simple method of estimating fifty per cent endpoints. Am J Hyg 27: 493-497.

Rosen L, Rozeboom LF, Gubler DJ, Kien JC, Chianotis BN 1985. Comparative susceptibility of mosquito species and strains to oral and parenteral infection with dengue and Japanese encephalitis viruses. Am J Trop Med Hyg 34: 603615.

Rosen L, Shroyer DA, Tesh RB, Freire JF, Ching Lien JIH 1983. Transovarial transmission of dengue viruses by mosquitos: Aedes albopictus and Aedes aegypti. Am J Trop Med Hyg 32: 1108-1119.

Santos RLC 2003. Atualização da distribuição de Aedes albopictus no Brasil (1997-2002). Rev Saúde Pública 37: 671-673. 
Schatzmayr HG 2000. Dengue situation in Brazil by year 2000. Mem Inst Oswaldo Cruz, 95: 179-181.

Serufo JC, Oca HM, Tavares AV, Souza AM, Rosa RV, Jamal MC, Lemos JR, Oliveira MA, Nogueira RMR, Schatzmayr HG 1993. Isolation of dengue virus type 1 from larvae of Aedes albopictus in Campos Altos city, state of Minas Gerais, Brazil. Mem Inst Oswaldo Cruz 88: 503-504.

Shroyer DA 1986. Aedes albopictus and arboviruses: a concise review of the literature. J Am Mosq Control Assoc 2: 424428.

Sunarto J, Gubler DJ, Nalim S, Eram S, Saroso-Sulianti J 1979. Epidemic dengue hemorrhagic fever in rural Indonésia. Am J Trop Med Hyg 28: 717-724.
Vazeille M, Rosen L, Mousson L, Failloux AB 2003. Low oral receptivity for dengue type 2 viruses of Aedes albopictus from southeast Asia compared with that of Aedes aegypti. Am J Trop Med Hyg 68: 203-208.

Vazeille-Falcoz M, Mousson L, Rodhain F, Chungue E, Failloux AB 1999. Variation in oral susceptibility to dengue type 2 virus of populations os Aedes aegypti from the island of Tahiti and Morea, French Polynesia. Am J Trop Med Hyg 60: 292-299.

Woodring JL, Higgs S, Beaty BJ 1996. Natural cycles of vectorborne pathogens. In BJ Beaty, WC Marquardt (eds), The Biology of Disease Vectors, University Press of Colorado, Niwot, $632 \mathrm{pp}$. 